\title{
Fleshy fungi of the genera Armillaria, Pleurotus, and Grifola as habitats of Coleoptera
}

\author{
DMITRY S. SCHIGEL
}

\begin{abstract}
SCHIGEL, D. S. 2007: Fleshy fungi of the genera Armillaria, Pleurotus, and Grifola as habitats of Coleoptera. - Karstenia 47: 00-00. Helsinki. ISSN 0453-3402.

The paper deals with adult beetles attracted by wood-rotting agarics of the genera Armillaria and Pleurotus, plus those collected on the oak-decaying polypore Grifola frondosa. The interrelations of 14 species (110 samples) of fungi and 78 species (1691 individuals) of beetles are discussed. More close relationships, such as fungivory of larvae of Coleoptera are also treated. In southern Finland larvae of Cychramus (in Armillaria) and Triplax (in Pleurotus) are able to utilize fleshy wood-rotting fungi. The majority of beetles were recorded as adults, including $52(67 \%)$ species of Staphylinidae.
\end{abstract}

Key words: Basidiomycota, Coleoptera, decay, ecology, Finland, fungivory.

Dmitry S. Schigel, Botanical Museum, Finnish Museum of Natural History, P.O. Box 7, FI-00014 University of Helsinki, Finland.E-mail: dmitry.shchigel@helsinki.fi

\section{Introductio}

Woody plants make most of the forest biomass, and, alive or dead, become eventually utilized by various organisms, including insects and fungi. Polypores are the main group of wood-decomposing fungi especially at early stages of wood decay. Many agarics continue the decay process at later stages. Polypores usually have sturdy and long-lasting fruit bodies, and even the softest species last in nature longer than water-rich agarics. This has consequences on insect fauna: beetles are diverse on polypores, while diptera larvae mostly utilize mushrooms.

My field work in the autumn 2006 coincided with the peak fruiting time of Armillaria and ample material of beetles could be harvested. The present paper focuses on beetles linked with Armillaria borealis Marxm. \& Korhonen. My goal was to explore the similarity of beetle communities on wood-rotting agarics in different conditions, in order to study the dependence of beetles on host species, and to review their life strategies and adaptations in comparison to polypores of southern Finland.

Previously six consistency classes of fungal fruit bodies could be separated among polypores
(Schigel et al. 2004), and the role of substrate rarity (Schigel \& Toresson 2005), and the seasonal dynamics of the attracted beetles (Schigel et al. 2006) were studied. The treatment would be incomplete without a report on Coleoptera found on wood-rotting fleshy fungi.

\section{Materials and methods}

Armillaria borealis and associated Coleoptera were collected during a polypore inventory in the Kolovesi National Park, southern Finland (Fig. 1). The study was organized by the Finnish Forest and Park Service (Metsähallitus) in September 2006. Supplementary materials from other agaric genera derive from other collecting trips to southern Finland in 2001-2006. These include the Åland Islands, Helsinki, the Ruissalo oak forest close to Turku, and several locations in Etelä-Häme (Table 1). All material was collected in the southern boreal zone (sensu Ahti et al. 1968) of Finland.

The Kolovesi National Park lies in the forested archipelago of Saimaa, one of the largest 


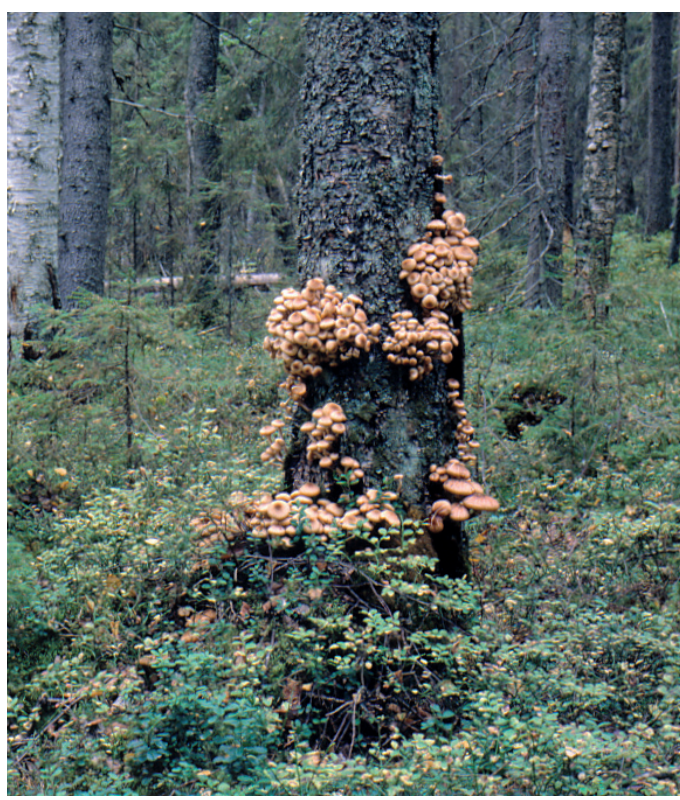

Fig. 1. Clusters of Armillaria borealis fruit bodies on birch, Kolovesi National Park, Pohjois-Savo. Photo T. lakes in Finland. The landscape is strongly influenced by the Ice Age and is dominated by cliff walls facing the lake, rugged terrain, and forested depressions along the lakesides, favoured by beavers. The woody vegetation is dominated by Norway spruce (Picea abies), birch species (Betula spp.), aspen (Populus tremulal) and Scots pine (Pinus sylvestris). Rock outcrops are covered by pine woodlands interrupted with small spruce swamps, and to a minor extent herb-rich forests. The high volume of dead wood increases the conservation value of these fairly old forests, more than a half of them being over one hundred years of age.

All fungal specimens were collected for identification, and dried in mushroom dryers with ventilated air at $+40-45^{\circ} \mathrm{C}$. In difficult cases provisional names were later approved by microscopic study of sections mounted in Cotton Blue or Melzer's reagent at $\times 1250$ magnification and phase contrast illumination. Fungal collections are preserved in the Herbarium of the Botanical Museum, Finnish Museum of

Table 1. Finnish localities mentioned in this paper.

\begin{tabular}{|c|c|c|c|c|c|}
\hline Code & Locality & Commune & Province & Coordinat & \\
\hline$\AA$ & Åland Islands & several & Ahvenanmaa & $\mathrm{N} 60^{\circ}{ }^{\prime}$ & E $19^{\circ} 58^{\prime}$ \\
\hline $\mathrm{H}$ & Helsinki & Helsinki & Uusimaa & N $60^{\circ} 12^{\prime}$ & E $24^{\circ} 57^{\prime}$ \\
\hline $\mathrm{Kt}$ & Kotinen Virgin Forest & Lammi & Etelä-Häme & $\mathrm{N} 61^{\circ} 14^{\prime}$ & E $25^{\circ} 3^{\prime}$ \\
\hline $\mathrm{Kv}$ & Kolovesi National Park & Enonkoski & Pohjois-Savo & $\mathrm{N} 62^{\circ} 15^{\prime}$ & E $28^{\circ} 49^{\prime}$ \\
\hline $\mathrm{L}$ & Lammi Biological Station & Lammi & Etelä-Häme & N 613', & E $25^{\circ} 2^{\prime}$ \\
\hline $\mathrm{R}$ & Ruissalo oak forest & Turku & Varsinais-Suomi & $\mathrm{N} 60^{\circ} 25^{\prime}$ & E $22^{\circ} 10^{\prime}$ \\
\hline $\mathrm{S}$ & Susimäki Virgin Forest & Ruovesi & Etelä-Häme & $\mathrm{N} 61^{\circ} 51^{\prime}$ & E $24^{\circ} 14^{\prime}$ \\
\hline
\end{tabular}

Natural History, University of Helsinki (H); their nomenclature follows Salo et al. (2005) and Niemelä (2005).

Clusters of fruit bodies were studied for beetles. Adult beetles were collected and counted separately from larvae. All adult beetles and samples of larvae were preserved in 70\% alcohol for identification. Fungi which contained additional beetle larvae were removed and placed for rearing in plastic 0.5-litre containers. Each container was quarter-filled with gardening peat to serve for pupation media. Perforated lids allowed slow drying of the fruit bodies. Rearing chambers were not moistened during the rear- ing. Collections made in summer-autumn were kept for 2-3 months in outdoor temperature, then exposed to room temperature for further two months, and after that checked for insects. Beetles are preserved in the Finnish Museum of Natural History, University of Helsinki; single individuals have been given to identifiers (see Acknowledgements).

Beetle names accord Silfverberg (2004). Authors of the Latin names are omitted from the text, but are shown in Table 2 for fungi and Table 3 for beetles. Terminology follows the previous papers in the series (Schigel et al. 2004, 2006, Schigel \& Toresson 2005). 


\section{Results}

76 forest compartments (metsäkuviot) were surveyed for polypores, and yielded 98 species of polypores and 48 species of other fungi (Niemelä $\&$ Kinnunen 2006). In the present paper the interrelations of 14 species (110 samples) of fungi and 78 species (1691 individuals) of beetles are discussed. It comes as no surprise that as many as $52(67 \%)$ beetle species in the report belong to the Staphylinidae, as they usually outnumber the other beetles in gilled fungi (Table 2). All beetles were sampled as adults, and for Cychramus and Triplax also larvae were recorded. Four kinds of fleshy wood-rotting fungi were sampled for Coleoptera:

(1) Among the three species of long-stiped agarics studied for associated beetles, the most diverse community (13 species) was found on Megacollybia platyphylla (Table 2). The bee-

Table 2. Arimllaria borealis plus selected other wood-rotting fungi, and their beetles in Southern Finland. Species of fungi are given in an alphabetical order; IUCN threat categories (Rassi et al. 2001) are shown. I/S = number of fungal specimens inhabited by beetles / number of fungal specimens studied. Numbers of insect specimens found or reared are given in parentheses after the beetle species name. Adult insects collected in nature indicated in light italics; larvae or reared adults (beetle - fungus associations meeting the Lawrence (1973) criterion) in bold italics.

\begin{tabular}{|c|c|c|c|}
\hline \# & Fungus species & $\mathrm{I} / \mathrm{S}$ & Insect records \\
\hline 1 & $\begin{array}{l}\text { Armillaria borealis } \\
\text { Marxm. \& Korhonen }\end{array}$ & $665 / 38$ & 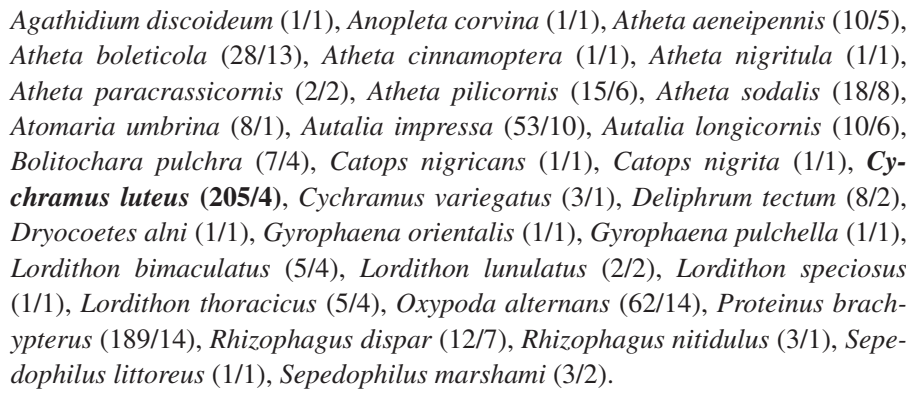 \\
\hline 2 & $\begin{array}{l}\text { Crepidotus calolepis } \\
\text { (Fr.) P. Karst. }\end{array}$ & $8 / 2$ & Deliphrum tectum (1/1), Proteinus brachypterus $(7 / 1)$. \\
\hline 3 & $\begin{array}{l}\text { Hygrophoropsis auran- } \\
\text { tiaca (Wulfen : Fr.) J. } \\
\text { Schröt. }\end{array}$ & $6 / 2$ & $\begin{array}{l}\text { Atheta pilicornis (1/1), Autalia longicornis (1/1), Gyrophaena bihamata (1/1), } \\
\text { Proteinus brachypterus (3/1). }\end{array}$ \\
\hline 4 & $\begin{array}{l}\text { Hypsizygus ulmarius } \\
\text { (Bull. : Fr.) Redhead }\end{array}$ & $44 / 4$ & Atomaria affinis (35/5), Cerylon ferrugineum (4/1), Rhizophagus dispar (5/2). \\
\hline 5 & $\begin{array}{l}\text { Lentinellus castoreus } \\
\text { (Fr.) Kühner \& Maire }\end{array}$ & $9 / 3$ & $\begin{array}{l}\text { Acrulia inflata }(2 / 1), \text { Agathidium discoideum }(3 / 1) \text {, Atheta nigritula }(1 / 1), \text { Atheta } \\
\text { pilicornis }(1 / 1), \text { Rhizophagus dispar }(1 / 1), \text { Sepedophilus marshami }(1 / 1) \text {. }\end{array}$ \\
\hline 6 & $\begin{array}{l}\text { Lentinellus vulpinus } \\
\text { (Sowerby : Fr.) Kühner } \\
\text { \& Maire }\end{array}$ & $4 / 1$ & $\begin{array}{l}\text { Acrulia inflata (1/1), Atheta crassicornis (1/1), Atheta pilicornis (1/1), Rhizopha- } \\
\text { gus dispar (1/1). }\end{array}$ \\
\hline 7 & $\begin{array}{l}\text { Megacollybia platy- } \\
\text { phylla (Pers. : Fr.) Kotl. } \\
\text { \& Pouzar }\end{array}$ & $140 / 7$ & $\begin{array}{l}\text { Atheta aeneipennis }(2 / 1), \text { Atheta boleticola }(14 / 3) \text {, Atheta paracrassicornis }(1 / 1) \text {, } \\
\text { Atheta pilicornis }(3 / 2) \text {, Atheta sodalis }(1 / 1) \text {, Autalia impressa }(3 / 1) \text {, Autalia } \\
\text { longicornis }(2 / 2), \text { Bolitochara pulchra }(1 / 1), \text { Catops nigrita }(1 / 1), \text { Lordithon tho- } \\
\text { racicus }(3 / 1), \text { Oxypoda alternans }(1 / 1) \text {, Proteinus brachypterus }(107 / 4) \text {, Sepedo- } \\
\text { philus marshami }(1 / 1) \text {. }\end{array}$ \\
\hline
\end{tabular}




\begin{tabular}{|c|c|c|c|}
\hline \# & Fungus species & $\mathrm{I} / \mathrm{S}$ & Insect records \\
\hline 8 & $\begin{array}{l}\text { Panellus serotinus } \\
\text { (Schrad. : Fr.) J.G. } \\
\text { Kühn }\end{array}$ & $1 / 1$ & Acrulia inflata (1/1). \\
\hline 9 & $\begin{array}{l}\text { Pholiota squarrosoides } \\
\text { (Peck) Sacc. }\end{array}$ & $2 / 1$ & Cerylon ferrugineum (1/1), Gnathoncus nannetensis (1/1). \\
\hline 10 & $\begin{array}{l}\text { Pleurotus dryinus } \\
\text { (Pers. : Fr.) P. Kumm }\end{array}$ & $40 / 6$ & $\begin{array}{l}\text { Atheta castanoptera (1/1), Atomaria affinis (1/1), Cryptophagus scanicus }(5 / 2) \text {, } \\
\text { Rhizophagus bipustulatus }(1 / 1), \text { Rhizophagus dispar }(2 / 1) \text {, Rhizophagus nitidulus } \\
(1 / 1) \text {, Sepedophilus testaceus }(1 / 1) \text {. }\end{array}$ \\
\hline 11 & $\begin{array}{l}\text { Pleurotus ostreatus } \\
\text { (Jacq. : Fr.) P. Kumm. }\end{array}$ & $28 / 2$ & Cerylon ferrugineum (1/1), Triplax aenea (27/2). \\
\hline 12 & $\begin{array}{l}\text { Pleurotus pulmonarius } \\
\text { (Fr.) Quél. }\end{array}$ & $423 / 31$ & $\begin{array}{l}\text { Agathidium confusum }(1 / 1) \text {, Agathidium pisanum }(1 / 1) \text {, Atheta aeneipennis } \\
(14 / 3) \text {, Atheta boleticola }(1 / 1) \text {, Atheta boletophila }(1 / 1) \text {, Atheta crassicornis }(2 / 2) \text {, } \\
\text { Atheta pallidicornis }(2 / 1) \text {, Atheta paracrassicornis }(2 / 1) \text {, Atheta picipes }(13 / 5) \text {, } \\
\text { Atheta pilicornis }(1 / 1) \text {, Atheta sodalis }(2 / 2) \text {, Atheta subtilis }(20 / 5) \text {, Atomaria af- } \\
\text { finis }(1 / 1) \text {, Autalia impressa }(8 / 1) \text {, Autalia longicornis }(18 / 2) \text {, Cerylon ferrugi- } \\
\text { neum }(4 / 2) \text {, Cryptophagus confusus }(1 / 1) \text {, Deliphrum tectum }(9 / 1) \text {, Endomychus } \\
\text { coccineus }(2 / 1) \text {, Glischrochilus hortensis }(7 / 1) \text {, Gyrophaena affinis }(1 / 1) \text {, Gy- } \\
\text { rophaena angustata }(6 / 3) \text {, Gyrophaena fasciata }(62 / 2) \text {, Gyrophaena joyi }(7 / 1) \text {, Gy- } \\
\text { rophaena orientalis }(1 / 1) \text {, Gyrophaena poweri }(1 / 1) \text {, Gyrophaena strictula }(4 / 2) \text {, } \\
\text { Leptusa pulchella }(5 / 2) \text {, Lordithon lunulatus }(4 / 4) \text {, Lordithon speciosus }(6 / 3) \text {, } \\
\text { Lordithon thoracicus }(2 / 2) \text {, Lordithon trimaculatus }(10 / 2) \text {, Megarthrus depressus } \\
\text { (2/1), Omalium rivulare }(2 / 2) \text {, Oxypoda alternans }(32 / 1) \text {, Proteinus brachypterus } \\
(60 / 3) \text {, Quedius plagiatus }(1 / 1) \text {, Rhizophagus dispar }(42 / 8) \text {, Rhizophagus nitidu- } \\
\text { lus }(1 / 1) \text {, Sciodrepoides watsoni }(1 / 1) \text {, Tachinus laticollis }(3 / 2) \text {, Tachinus pallipes } \\
\text { (2/1), Triplax aenea }(31 / 6) \text {, Triplax rufipes }(23 / 5)\end{array}$ \\
\hline 13 & $\begin{array}{l}\text { Volvariella bombycina } \\
\text { (Schaeff. : Fr.) Singer }\end{array}$ & $76 / 4$ & $\begin{array}{l}\text { Cerylon histeroides }(2 / 1), \text { Gyrophaena affinis }(2 / 1) \text {, Gyrophaena bihamata }(4 / 2) \text {, } \\
\text { Gyrophaena congrua }(1 / 1) \text {, Gyrophaena fasciata }(2 / 1) \text {, Gyrophaena joyioides } \\
(58 / 3) \text {, Lordithon thoracicus }(4 / 1) \text {, Pocadius ferrugineus }(3 / 1) \text {. }\end{array}$ \\
\hline 14 & $\begin{array}{l}\text { Grifola frondosa (J. } \\
\text { Dicks.: Fr.) Gray, VU }\end{array}$ & $245 / 8$ & $\begin{array}{l}\text { Aleochara stichai }(2 / 1), \text { Atheta aeneipennis }(1 / 1) \text {, Atheta castanoptera }(4 / 1) \text {, } \\
\text { Atheta crassicornis }(64 / 1), \text { Atheta gagatina }(1 / 1) \text {, Atheta marcida }(3 / 2) \text {, Atheta } \\
\text { nigritula }(29 / 1) \text {, Atheta paracrassicornis }(65 / 1) \text {, Atheta picipes }(2 / 1) \text {, Atheta pili- } \\
\text { cornis }(5 / 1) \text {, Atheta sodalist }(5 / 1) \text {, Autalia longicornis }(1 / 1) \text {, Catops coracinus } \\
(5 / 2), \text { Deliphrum tectum }(12 / 1), \text { Lordithon bimaculatus }(1 / 1), \text { Lordithon lunulatus } \\
(23 / 1) \text {, Lordithon thoracicus }(2 / 1) \text {, Omalium rivulare }(4 / 1) \text {, Philhygra malleus } \\
(1 / 1), \text { Philonthus fimetarius }(2 / 1), \text { Philorhizus sigma }(1 / 1), \text { Proteinus brachypter }- \\
\text { us }(9 / 1) \text {, Sepedophilus testaceus }(1 / 1) \text {, Tachinus proximus }(2 / 1)\end{array}$ \\
\hline
\end{tabular}

tle Proteinus brachypterus dominated (76\% of total individuals sampled from the fungus) and was found on every studied long-stiped fungus. The second-commonest species (10\%) Atheta boleticola was found along with other, mostly Staphylinidae species represented by 1-2 individuals each. These include Atheta species and larger Staphylinidae, such as Lordithon and Sepedophilus. In the mushroom Hygrophoropsis aurantiaca four associated beetle species were registered, two of which (Proteinus brachypter$u s$ and Autalia longicornis) were found also on Megacollybia. Unlike these two fungus species, Volvariella bombycina was characterized by five Gyrophaena species, of which G. joyioides dominated $(76 \%$ of total individuals sampled from the fungus). Gyrophaena bihamata is the only species found on both Volvariella and Hygrophoropsis (Table 2 ). 


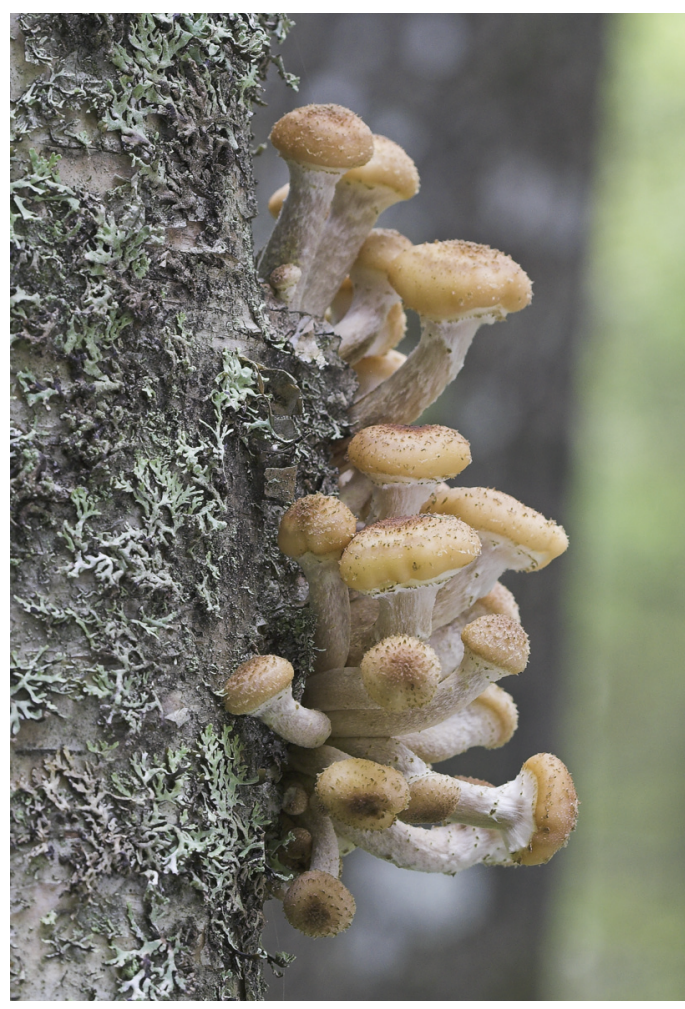

Fig. 2. Young fruit bodies of Armillaria borealis on birch snag, Kolovesi National Park, Pohjois-Savo. Photo D. Schigel, 2006.

(2) In Kolovesi clusters of Armillaria borealis (Fig. 2) were growing simultaneously on different altitudes above ground. The moister and lower-located clusters were first to disappear, but richest in beetles. Clusters over $0.5 \mathrm{~m}$ above ground hosted only single individuals of Cerylon, in spite of their slightly extended lifetime, and clusters above $2 \mathrm{~m}$ were in most cases intact and free from beetles. Larvae of Cychramus spp. are able to utilize the interior of short-living fruit bodies of Armillaria. Two Cychramus species were registered on Armillaria borealis - countless swarms of the common C. luteus in Kolovesi and Helsinki, and the more rare $C$. varieagtus in Susimäki (Figs. 3, 4). Adult Cychramus luteus were dominating on younger Armillaria fruit bodies in Helsinki, and only a few Staphylinidae were observed. The reverse was seen on mature fruit bodies of Armillaria in Kolovesi: Proteinus brachypterus $(41 \%$ of total individuals sampled from the fungus) were the most common visitors,

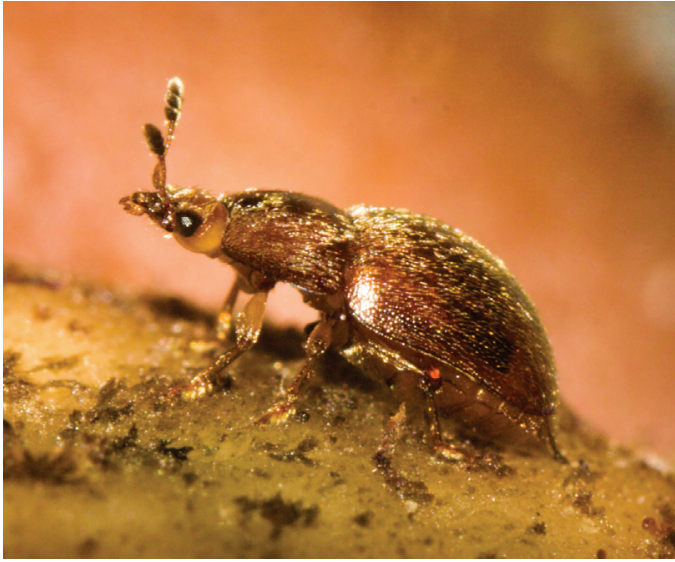

Fig. 3. Adult Cychramus luteus to colonize juvenile fruit body of Armillaria borealis, Kumpula, Helsinki. Photo D. Schigel, 2007.

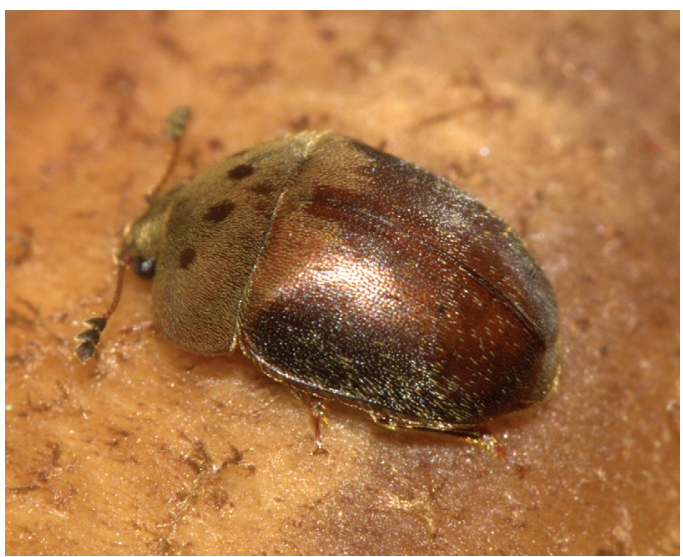

Fig. 4. Adult Cychramus variegatus on the cap of Armillaria borealis, Susimäki Virgin Forest, Etelä-Häme. Photo D. Schigel, 2005.

followed by Oxypoda alternans (13\%), Autalia impressa (12\%), and Atheta boleticola (6\%). Less frequent were other Staphylinidae, including six more species of Atheta, and two species of Sepedophilus. Agathidium discoideum, Atomaria umbrina, two species of Catops and two of Rhizophagus contributed to the family and ecological diversity of the associated species set, a total of 31 species (Table 2).

(3) Among the log-favouring pleurotoid fungi, Crepidotus calolepis, Panellus serotinus, Lentinellus castoreus, and L. vulpinus were visited 
by generalists such as Proteinus brachypterus and Acrulia inflata. Unlike other fungi of the kind, Hypsizygus ulmarius was ignored by adult Staphylinidae, but favoured by Atomaria affinis. Rhizophagus dispar, numerous on Pleurotus pulmonarius (Fig. 5) and several polypore species, was spotted on the majority of the studied pleurotoid fungi. Similarly to Hypsizygus ulmarius, Pleurotus dryinus and P. pulmonarius attracted Cryptophagidae, such as Atomaria affinis, Cryptophagus scanicus and C. confusus. Fruit bodies of Pleurotus pulmonarius hosted 44 beetle species - the highest number in this study. Of these, dominating visitors were Gyrophaena fasciata (15\% of total individuals sampled from the fungus), Proteinus brachypterus (14\%), Oxypoda alternans (8\%) and Atheta aeneipennis (3\%). The list of visitors includes nine Atheta, six Gyrophaena, and four Lordithon species.

(4) Of the total 24 beetle species associated with the red-listed (Rassi et al. 2001) polypore Grifola frondosa (Fig. 6), the most typical beetles were Atheta paracrassicornis $(27 \%$ of total individuals sampled from the fungus) and A. crassicornis (26\%), with frequent Lordithon lunulatus (9\%). The less numerous species were mainly Staphylinidae, including eight other species of Atheta.

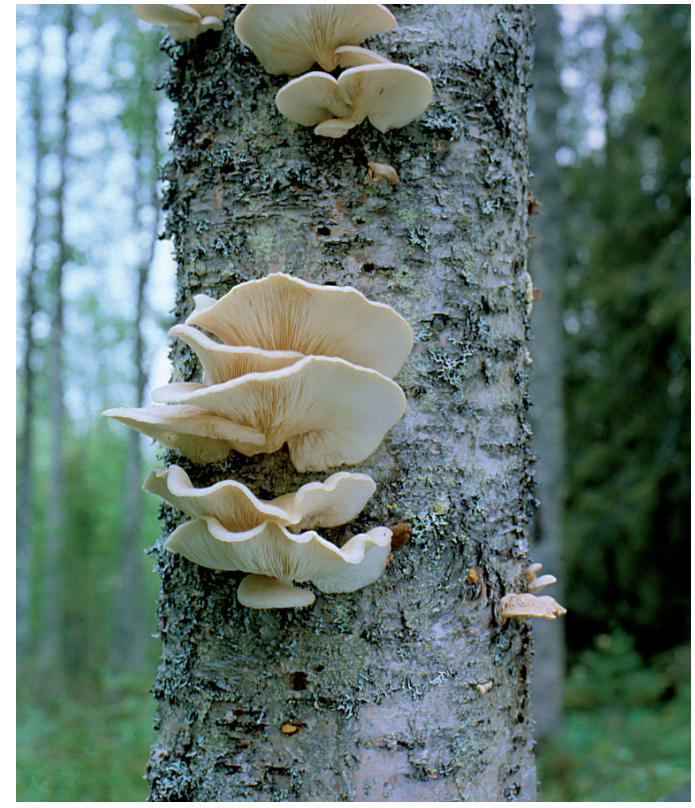

Fig. 5. Pleurotus pulmonarius on birch, Aakenustunturi, Kittilän Lappi. Photo T. Niemelä, 2000.

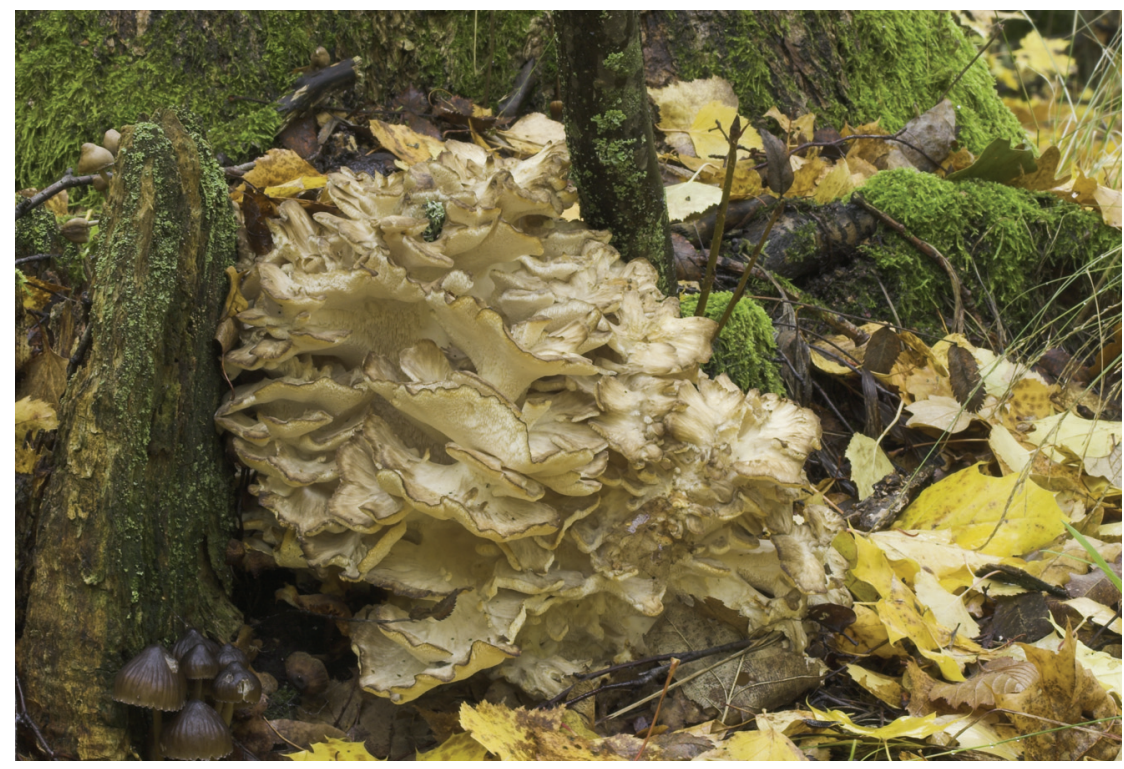

Fig. 6. Grifola frondosa at the base of oak stump in Ruissalo forest, Turku. Photo D. Schigel, 2004. 
Table 3. Systematic list of beetles attracted to Armillaria borealis and selected other wood-rotting fungi in southern Finland. FS/IUCN = Frequency score of beetles (Rassi 1993) / threat categories (Rassi et al. 2001), if relevant; threat categories and scores of the uncommon and rare beetle species in Finland (30 points and higher) are indicated in bold face; Loc = locality, for details see Table 1: $\AA$ $=$ Åland Islands, $\mathrm{H}=$ Helsinki, Kt = Kotinen Virgin Forest, Etelä-Häme, Kv = Kolovesi National Park, Pohjois-Savo, L = Lammi Biological Station, Etelä-Häme, R = Ruissalo Island, Varsinais-Suomi, S $=$ Susimäki Virgin Forest, Etelä-Häme. Numbers refer to the species of fungi (Table 2); light face = records of adult beetles; bold face $=$ larvae or rearings .

\begin{tabular}{|c|c|c|c|}
\hline Taxon & FS/IUCN & Loc & Host fungi \\
\hline \multicolumn{4}{|l|}{ COLEOPTERA } \\
\hline \multicolumn{4}{|l|}{ Carabidae Latreille, 1802} \\
\hline Philorhizus sigma (Rossi, 1790) & 4 & $\mathrm{R}$ & 14 \\
\hline \multicolumn{4}{|l|}{ Histeridae Gyllenhal, 1808} \\
\hline Gnathoncus nannetensis (Marseul, 1862) & 20 & $\mathrm{Kt}$ & 9 \\
\hline \multicolumn{4}{|l|}{ Leiodidae Fleming, 1821} \\
\hline Agathidium confusum Brisout de Barneville, 1863 & 6 & Kt & 12 \\
\hline Agathidium discoideum Erichson, 1845 & 40 & $\mathrm{Kv}$ & 1,5 \\
\hline Agathidium pisanum Brisout de Barneville, 1872 & 10 & $\mathrm{Kt}$ & 12 \\
\hline Catops coracinus Kellner, 1846 & 10 & $\mathrm{R}$ & 14 \\
\hline Catops nigricans (Spence, 1815) & 10 & $\mathrm{Kv}$ & 1 \\
\hline Catops nigrita Erichson, 1837 & 2 & $\mathrm{Kv}$ & 1,7 \\
\hline Sciodrepoides watsoni (Spence, 1815) & 1 & $\mathrm{~L}$ & 12 \\
\hline \multicolumn{4}{|l|}{ Staphylinidae Latreille, 1802} \\
\hline Acrulia inflata (Gyllenhal, 1813) & 6 & $\mathrm{Kv}$ & $5,6,8$ \\
\hline Aleochara stichai Likovský, 1965 & 40 & $\mathrm{R}$ & 14 \\
\hline Anopleta corvina (Thomson, 1856) & 15 & $\mathrm{Kv}$ & 1 \\
\hline Atheta aeneipennis (Thomson, 1856) & 2 & $\mathrm{~L}, \mathrm{Kt}, \mathrm{Kv}, \mathrm{R}$ & $1,7,12,14$ \\
\hline Atheta boleticola J.Sahlberg, 1876 & 15 & $\mathrm{Kt}, \mathrm{Kv}$ & $1,7,12$ \\
\hline Atheta boletophila (Thomson, 1856) & 30 & $\mathrm{R}$ & 12 \\
\hline Atheta castanoptera (Mannerheim, 1830) & $100 / \mathrm{VU}$ & $\AA, \mathrm{R}$ & 10,14 \\
\hline Atheta cinnamoptera (Thomson, 1856) & 2 & $\mathrm{Kv}$ & 1 \\
\hline Atheta crassicornis (Fabricius, 1793) & 4 & $\mathrm{Kv}, \mathrm{L}, \mathrm{R}$ & $6,12,14$ \\
\hline Atheta gagatina (Baudi, 1848) & 6 & $\mathrm{R}$ & 14 \\
\hline Atheta marcida (Erichson, 1837) & $100 / \mathrm{NT}$ & $\mathrm{R}$ & 14 \\
\hline Atheta nigritula (Gravenhorst, 1802) & 10 & $\mathrm{Kv}, \mathrm{R}$ & $1,5,14$ \\
\hline Atheta pallidicornis (Thomson, 1856) & 40 & $\mathrm{Kt}$ & 12 \\
\hline Atheta paracrassicornis Brundin, 1954 & 3 & $\mathrm{~L}, \mathrm{Kv}, \mathrm{R}$ & $1,7,12,14$ \\
\hline Atheta picipes (Thomson, 1856) & 15 & $\mathrm{R}$ & 12,14 \\
\hline Atheta pilicornis (Thomson, 1852) & 10 & $\mathrm{Kt}, \mathrm{Kv}, \mathrm{R}$ & $1,3,5,7,12,14$ \\
\hline Atheta sodalis (Erichson, 1837) & 2 & $\mathrm{~L}, \mathrm{Kt}, \mathrm{Kv}, \mathrm{R}$ & $1,7,12,14$ \\
\hline Atheta subtilis (Scriba, 1866) & 1 & $\mathrm{~L}, \mathrm{Kt}, \mathrm{R}$ & 12 \\
\hline Autalia impressa (Olivier, 1795) & 60 & $\mathrm{Kt}, \mathrm{Kv}$ & $1,7,12$ \\
\hline Autalia longicornis Scheerpeltz, 1947 & 4 & $\mathrm{~L}, \mathrm{Kt}, \mathrm{Kv}, \mathrm{R}$ & $1,3,7,12,14$ \\
\hline Bolitochara pulchra (Gravenhorst, 1806) & 2 & $\mathrm{Kv}$ & 1,7 \\
\hline Deliphrum tectum (Paykull, 1789) & 1 & $\mathrm{Kt}, \mathrm{Kv}$ & $1,2,12,14$ \\
\hline Gyrophaena affinis Mannerheim, 1830 & 2 & $\mathrm{H}, \mathrm{Kt}$ & 12,13 \\
\hline Gyrophaena angustata (Stephens, 1832) & 30 & $\mathrm{R}$ & 12 \\
\hline Gyrophaena bihamata Thomson, 1867 & 15 & $\mathrm{H}, \mathrm{Kt}$ & 3,13 \\
\hline Gyrophaena congrua Erichson, 1837 & 30 & $\mathrm{H}$ & 13 \\
\hline Gyrophaena fasciata (Marsham, 1802) & 2 & $\mathrm{H}$ & 12,13 \\
\hline Gyrophaena joyi Wendeler, 1924 & 40 & $\mathrm{Kt}$ & 12 \\
\hline Gyrophaena joyioides Wüsthoff, 1937 & 10 & $\mathrm{H}$ & 13 \\
\hline Gyrophaena orientalis Strand, 1938 & 40 & $\mathrm{Kt}$ & 1,12 \\
\hline Gyrophaena pulchella Heer, 1839 & 15 & $\mathrm{Kv}$ & 1 \\
\hline
\end{tabular}




\begin{tabular}{|c|c|c|c|}
\hline Taxon & FS/IUCN & Loc & Host fungi \\
\hline Gyrophaena poweri Crotch, 1866 & 10 & $\mathrm{Kt}$ & 12 \\
\hline Gyrophaena strictula Erichson, 1839 & 15 & $\mathrm{R}$ & 12 \\
\hline Leptusa pulchella (Mannerheim, 1830) & 1 & $\mathrm{R}$ & 12 \\
\hline Lordithon lunulatus (Linnaeus, 1761) & 2 & $\mathrm{Kv}, \mathrm{R}$ & $1,12,14$ \\
\hline Lordithon speciosus (Erichson, 1840) & 20 & $\mathrm{Kv}$ & 1,12 \\
\hline Lordithon thoracicus (Fabricius, 1777) & 1 & $\mathrm{H}, \mathrm{Kt}, \mathrm{Kv}, \mathrm{R}$ & $1,7,12,13,14$ \\
\hline Lordithon trimaculatus (Fabricius, 1793) & 30 & $\mathrm{Kt}$ & 12 \\
\hline Lordithon trinotatus (Erichson,1839) & $100 / \mathrm{NT}$ & $\mathrm{Kv}, \mathrm{R}$ & 1,14 \\
\hline Megarthrus depressus (Paykull, 1789) & 6 & $\mathrm{~L}$ & 12 \\
\hline Omalium rivulare (Paykull, 1789) & 1 & $\mathrm{Kt}, \mathrm{R}$ & 12,14 \\
\hline Oxypoda alternans (Gravenhorst, 1802) & 2 & $\mathrm{Kt}, \mathrm{Kv}$ & $1,7,12$ \\
\hline Philhygra malleus (Joy, 1913) & 6 & $\mathrm{R}$ & 14 \\
\hline Philonthus fimetarius (Gravenhorst, 1802) & 6 & $\mathrm{R}$ & 14 \\
\hline Proteinus brachypterus (Fabricius, 1792) & 1 & $\mathrm{~L}, \mathrm{Kt}, \mathrm{Kv}, \mathrm{R}$ & $1,2,3,7,12,14$ \\
\hline Quedius plagiatus Mannerheim, 1843 & 2 & $\mathrm{Kt}$ & 12 \\
\hline Sepedophilus littoreus (Linnaeus, 1758) & 4 & $\mathrm{Kv}$ & 1 \\
\hline Sepedophilus marshami (Stephens, 1832) & 6 & $\mathrm{Kv}$ & $1,5,7$ \\
\hline Sepedophilus testaceus (Fabricius, 1793) & 2 & $\mathrm{R}$ & 10,14 \\
\hline Tachinus laticollis Gravenhorst, 1802 & 2 & L, Kt & 12 \\
\hline Tachinus pallipes (Gravenhorst, 1806) & 1 & Kt & 12 \\
\hline Tachinus proximus Kraatz, 1855 & 2 & $\mathrm{R}$ & 14 \\
\hline \multicolumn{4}{|l|}{ Nitidulidae Latreille, 1802} \\
\hline Glischrochilus hortensis (Geoffroy, 1785) & 4 & $\mathrm{~L}$ & 12 \\
\hline Cychramus luteus (Fabricius, 1787) & 4 & $\mathrm{H}, \mathrm{Kv}, \mathrm{S}$ & 1 \\
\hline Cychramus variegatus (Herbst, 1792) & 15 & $\mathrm{~S}$ & 1 \\
\hline Pocadius ferrugineus (Fabricius, 1775) & 6 & $\mathrm{H}$ & 13 \\
\hline \multicolumn{4}{|l|}{ Monotomidae Laporte de Castelnau, 1840} \\
\hline Rhizophagus bipustulatus (Fabricius, 1793) & 6 & $\AA$ & 10 \\
\hline Rhizophagus dispar (Paykull, 1800) & 2 & $\mathrm{Kv}$ & $1,4,5,6,10,12$ \\
\hline Rhizophagus nitidulus (Fabricius, 1798) & 30 & $\AA, \mathrm{Kt}, \mathrm{Kv}$ & $1,10,12$ \\
\hline \multicolumn{4}{|l|}{ Cryptophagidae Latreille, 1802} \\
\hline Atomaria affinis (F.Sahlberg, 1834) & 30 & $\mathrm{~S}$ & $4,10,12$ \\
\hline Atomaria umbrina (Gyllenhal, 1827) & 10 & $\mathrm{Kv}$ & 1 \\
\hline Cryptophagus confusus Bruce, 1934 & 60 & $\mathrm{Kt}$ & 12 \\
\hline Cryptophagus scanicus (Linnaeus, 1758) & 6 & $\AA$ & 10 \\
\hline \multicolumn{4}{|l|}{ Erotylidae Latreille, 1802} \\
\hline Triplax aenea (Schaller, 1783) & 15 & $\AA, \mathrm{R}$ & 11,12 \\
\hline Triplax rufipes (Fabricius, 1781) & 40 & $\mathrm{R}$ & 12 \\
\hline \multicolumn{4}{|l|}{ Cerylonidae Billberg, 1820} \\
\hline Cerylon ferrugineum Stephens, 1830 & 4 & $\mathrm{R}$ & $4,9,11,12$ \\
\hline Cerylon histeroides (Fabricius, 1793) & 2 & $\mathrm{H}$ & 13 \\
\hline \multicolumn{4}{|l|}{ Endomychidae Leach, 1815} \\
\hline Endomychus coccineus (Linnaeus, 1758) & 10 & $\mathrm{Kt}$ & 12 \\
\hline \multicolumn{4}{|l|}{ Curculionidae Latreille, 1802} \\
\hline Dryocoetes alni (Georg, 1856) & 10 & $\mathrm{Kv}$ & 1 \\
\hline
\end{tabular}




\section{Discussion}

\subsection{Consistency classes of polypores and wood-rotting fleshy fungi}

The concept of consistency classes was created to describe and name the patterns of fungus-insect interactions found in nature (Schigel et al. 2004). This classification reflects the repeatedly found beetle species associations and the respective properties of host fungi (shape and volume of the fruit body, its durability, toughness, structure, water contents, and annuality $v s$. perenniality).

Different polypore fruit bodies were divided into six consistency classes. Perennial fruit bodies of fomitoid polypores (e.g. Phellinus, Fomitopsis, Ganoderma) attract beetles from the families Anobiidae, some Ciidae, and Tenebrionidae. Corky and leathery trametoid fruit bodies (e.g. Len- zites, Funalia, Cerrena) are favoured by species of Ciidae; tyromycetoid polypores (e.g. Postia, Amylocystis, Hapalopilus) are consumed by Hallomenus. Both piptoporoid and xanthocroic polypores produce annual, soft, voluminous and when living water-rich fruit bodies. In spite this similarity, their beetles are different: Tenebrionidae and Erotylidae mostly use piptoporoid polypores (e.g. Polyporus, Laetiporus), and Tetratomidae mostly xanthocroic ones (Inonotus, Onnia). Thin imbricate pilei of Trichaptum provide an environment for a few specialized beetle genera.

The course of changes within fruit body (juvenile - mature - old) differs between wood-rotting fleshy fungi and polypores. The first and main difference is the time scale: from days in agarics, to weeks or even years in polypores. Mature fruit bodies of agarics are always fertile and sporulating, which corresponds to polypore decomposi-

Table 4. Basidiocarp consistency classes among the of wood-rotting fleshy fungi, and examples of characteristic fungal and beetle genera in southern European taiga.

\footnotetext{
Agaricoid

Annual, prominently stipitate, thin, solitary or gregarious, usually on logs or stumps, extremely ephemeral and quickly decomposed into black slime.

Examples of fungal genera: Hygrophoropsis, Megacollybia, Volvariella.

Examples of beetles associated: adult Staphylinidae: Atheta pilicornis, Atheta boleticola, Autalia longicornis, Gyrophaena bihamata, Gyrophaena joyioides, Proteinus brachypterus.

NB: The extremely ephemeral nature of the fruit bodies and their erratic appearance in different years make agaricoid fungi unsuitable for beetle larvae, but visiting adults are numerous and diverse.
}

\section{Armillarioid}

Annual, prominently stipitate, thick and fibrous, usually with tens of fruit bodies packed in commonly rooted clusters, in lower parts of tree trunks and snags, ephemeral and decomposed into black slime, but in dry conditions dead fruit bodies persist over winter.

Examples of fungal genera: Armillaria, Pholiota.

Examples of beetles associated: Cychramus (including larvae), adult Staphylinidae.

NB: The voluminous and persistent wood-penetrating mycelium guarantees more regular fructification compared to the agaricoid ones. This predictable availability of the host allows Cychramus spp. to breed specifically in the fungus.

\section{Pleurotoid}

Annual, sessile or with rudimentary stipe, solitary or packed in commonly-rooted clusters of a few fruit bodies, on trunks or lower crown; fungus may continue to grow on downed trunks or fallen branches. Fruit bodies sometimes stay dried on the substrate after sporulation.

Examples of fungal genera: Crepidotus, Hypsizygus, Lentinellus, Panellus, Phyllotopsis, Pleurotus. Examples of beetles associated: Triplax (including larvae), adult Staphylinidae, Rhizophagus, and Cerylon.

NB: Similarly to armillarioid fruit bodies, pleurotoid ones host beetle larvae, which mostly finally drop to the soil for pupation (Triplax). Adults of Cerylon and Rhizophagus are repeatedly collected on gills of fully developed living basidiocarps. 
tion stage 2 (out of 4). Differently from agarics, the sporulation period of polypore fruit bodies is short compared with their lifetime. The decomposition processes of agarics and more robust polypores differ in the similar way: the fast collapse of the ephemeral fruit bodies of agarics and softer polypores requires swift larval development and escape for soil pupation, while the more durable polypores allow more lengthy larval development and possibility of pupation on the spot (decomposition stages 3 and 4).

\subsection{Agaricoid, armillarioid and pleurotoid consistency classes}

By analogy with polypores, three new consistency classes are needed to describe wood-rotting fleshy fungi: agaricoid, armillarioid and pleurotoid. These accord with the groups (1) to (3) in the previous section 3 (Results). Fruit bodies of these fungi differ from each other in their longevity, way of growth, location on tree trunk, and in the numbers of pilei per cluster. These consistency classes are described in Table 4 and below.

Agaricoid fungi are a diverse group of mostly solitary mushrooms of the forest floor and heavily decayed wood. These fungi show a variety of sizes and shapes, but their ephemeral habit and unclustered way of growth are the characteristic features. Beetles associated to agaricoid fungi are mainly generalist adult Staphylinidae. Even though only a few agaricoid species were documented for beetles in this study, it is likely that Staphylinidae will turn out to dominate in future collections from many other similar mushroom species.

Armillarioid consistency class by now comprises of two genera of fungi, Armillaria and Pholiota. The species-rich genus Pholiota is poorly documented for associated beetles. It seems, however, that Pholiota does not belong to the most commonly visited fungal substrates of generalist beetles. In Armillaria, like in many other fungi, the surface grazers (adult Coleoptera), and the fungivorous dwellers inside the moister fungal context (mostly larvae of Diptera) do not compete due to their spatial isolation. However, fungivorous larvae of dwellers sometimes reach high densities within the mushroom fruit bodies and then competitive interactions within and among species may occur. The extremely ephemeral nature of mushrooms causes the competition with time to be more critical for survival than the competition among individuals.

Co-occurrence of two Cychramus species on Armillaria was documented in Susimäki, with the unusual prevalence of $C$. variegatus. During the lab experiment adults of Cychramus luteus collected in Helsinki were eager to mate on the Armillaria fruit bodies. Probably, larvae of the two Cychramus species are able to develop in Armillaria, and as seen from the present Finnish pilot material, the prevalence of a certain species changes geographically. There is little doubt that larvae of Cychramus luteus in southern Finland dwell mainly in Armillaria fruit bodies, while more data on the biology of $C$. variegatus are needed. In addition to fungi, adults of these $C y$ chramus species are frequently collected on flowers during summertime, especially of the Apiaceae and Rosaceae. Cychramus luteus is feeding on pollen, not only on flowers, but also in bee hives, intruding to open the brood cells. Cychramus luteus does not otherwise affect brood, honey or combs, and was never found breeding there (Neumann \& Ritter 2004). The comparison of my data to the existing literature data is complicated by the fact that different Armillaria species were recognized only lately, and in most published studies they are collectively treated as "Armillaria mellea" - a species, that does not occur in Finland. Most probably, however, adult beetle visitors flexibly use the available Armillaria species, but the Cychramus species may be selective according to different geographical regions.

Pleurotoid fungi are found from the forest floor high up to branches, and they are more long-lasting than the other gilled wood-rotting mushrooms. Pleurotus may produce fruit bodies high in the tree crown, while others, like Crepidotus and Panellus mostly occupy logs and fallen branches. The most long-lasting, beetle-rich and best-studied fungi in this consistency class are the Pleurotus species, and probably the most interesting beetle genus in relation to Pleurotus is Triplax. In Nordic countries larvae of three Triplax species feed on Pleurotus fruit bodies. Still another, T. russica (Linnaeus, 1758) develops in Inonotus obliquus. Triplax russica and other beetle associates avoid the sterile conks of Inonotus obliquus, and come to colonize the sporulating fruit bodies as soon as the covering birch bark cracks. In the present material from southern Finland, two out of three Pleurotus-associated 
Triplax species were discovered. Triplax aenea and T. rufipes were reared in equal numbers from P. pulmonarius. Triplax aenea larvae also utilize P. ostreatus (Table 2).

Triplax scutellaris Charpentier, 1825, missing from this material, was earlier registered by me in Finnish Lapland (Pisavaara and Salla) colonizing Pleurotus pulmonarius and $P$. dryinus. This data overview shows the specificity of the three Triplax species to Pleurotus, but also their ability to flexibly use alternative host resources. Five adults of a rare, near-threatened species (Rassi et al. 1993, 2001), Agathidium pallidum (Gyllenhal, 1827), were collected from two samples of Pleurotus dryinus in eastern Finnish Lapland (Salla: Julmoiva) in 2005. Another Pleurotusdependent species, Cyllodes ater has now been rediscovered in eastern Finland (Yakovlev \& Hokkanen 1995). Beetles whose larvae develop rapidly in ephemeral substrates may have several generations a year depending on substrate availability. It is unclear if two or more generations of Triplax can complete their cycle within a single growth season in North Europe.

In Russian Far East Zaitsev and Kompantsev (1987) list Cyllodes, Triplax, and Eutriplax as obligate beetle dwellers whose larvae primarily feed on Pleurotus spp. These associations were detected also in other parts of Eurasia with such species as Triplax rubrica, T. collaris, T. lepida, and T. rufipes. Monophagous larvae of Triplax gracilenta are associated with East Asian Pleurotus citrinopileatus. Obligate inhabitants of Pleurotus in Russian Far East (Cyllodes, Triplax and Eutriplax) demonstrate how the life cycles are correlated with the longevity of fungal hosts. Larvae abandon decaying fruit bodies to pupate in soil. The pattern of colonisation and destruction is governed by host moisture: dry carpophores are mostly utilized by Coleoptera (Triplax, Eutriplax, Cyllodes), but wet ones are used by Diptera. Dry pilei of Pleurotus are similar in their consistency with piptoporoid polypores: Dacne larvae occupy Pleurotus, but also live in Piptoporus betulinus, Laetiporus sulphureus, and Spongipellis litschaueri. Very dry and hard fruit bodies of Pleurotus are durable enough to support larvae of Platydema dejeani and Ennearthron sp. (Zaitsev \& Kompantsev 1987).

Grifola frondosa - This oak-decaying polypore makes a special case as its way of growth and associated beetles resemble clustered wood- rotting agarics, rather than polypores. This fungus was observed in the Ruissalo oak forest. Late time of the fruit body growth combined with rarity may explain the absence of beetle larvae in Grifola frondosa. Voluminous clustered structure, fibrous fruit body, limited longevity, and a habit of growing at the base of oak trees make the fungus a meeting point for late-season adults of mycophilous Staphylinidae. Such ecological role of Grifola frondosa may be compared with Armillaria in Kolovesi.

\subsection{Beetles of wood-rotting agarics, a summary}

Certain parallelism in seasonality can be seen between wood-rotting mushrooms and polypores. Beetle larvae were absent from annual ephemeral polypores, and from agaricoid fungi. In annual sturdy polypores and pleurotoid agarics the peak of beetle activity fell on the living fruit bodies. Characteristics of armillarioid fungi are somewhat intermediate, and so is the set of associated beetles.

Differently from beetle-rich annual ephemeral and annual sturdy polypores, wood-rotting fleshy fungi hosted only the rapidly growing larvae of Cychramus and Triplax. These beetles are specific to only one host-fungus genus, Armillaria and Pleurotus, respectively. The structure and seasonality of fruit bodies of fleshy wood-rotting fungi make Staphylinidae the main group of adult beetle visitors. Typically one to three species of rove beetles outnumber the other species.

Annual fruit bodies of wood-rotting fungi provide beetles with an ecological fast-food service: these nutritional resources are often available for the visitors after they have hatched from pupae, but before their breeding hosts appeared. Only a few beetle species are adapted to use these ephemeral fungi as their breeding media.

\section{Conclusions}

Agaricoid, armillarioid, and pleurotoid fungi make three new consistency classes among the wood-rotting fungi. Together with polypores, discussed in previous papers of the series, fruit bodies of fleshy wood-rotting agarics provide a wide range of habitats for fungivorous Coleoptera, both adults and larvae. Our knowledge of fungivorous beetles and their host fungi has 
grown considerably, but some aspects of their ecology remain neglected. Among such topics is the impact of frost and drought on the seasonal dynamics of fungus-beetle systems. Ecologically frost and drought have a lot in common (periods of hibernation), but they require different physiological adaptations from fungi and beetles. Fruit bodies of ephemeral fungi can not resist extreme conditions; mycelium of some species react to a sudden change in temperature by starting to fruit in time unsuitable for beetle colonization, while perennial fruit bodies withstand repeated seasonal changes and attacks of beetles. I plan to expand my studies in fungus-beetle interactions into different climatic zones, and to other biogeographical aspects of the fungivory of Coleoptera, their life strategies and functional morphology.

The present pilot study covered fruit bodies of just a few wood-rotting fleshy fungi. Larvae of many beetle species depend on decayed wood penetrated by fungal mycelium. Other fungal substrates still require closer look: stereoid fungi may have similar substrate characteristics as the thin Trichaptum basidiocarps; boletes are probably visited by adult Staphylinidae generalist, like those on agarics. Ecology of beetles of both soft and sturdy Ascomycetes, the specialized inhabitants of Gasteromycetes, consumers of anamorphic fungi and fungi-like habitats such as Myxomycetes are to a large extent unknown.

\footnotetext{
Acknowledgements: Tuomo Niemelä kindly supervised the writing, and collaborated in the field together with Juha Kinnunen and Petri Oinonen, all from the University of Helsinki. Viktor B. Semenov (E. I. Marcinovsky Institute of Medical Parasitology and Tropical Medicine, Moscow), and Nikolay B. Nikitsky (Moscow State University) identified selected difficult beetle specimens. The PUTTE research programme for deficiently known and threatened forest species (Finnish Ministry of Environment) is thanked for supporting the study.
}

\section{References}

Ahti, T., Hämet-Ahti, L. \& Jalas, J. 1968: Vegetation zones and their sections in northwestern Europe. - Ann. Bot. Fennici 5: 169-211.

Lawrence, J.F. 1973: Host preference in Ciid beetles (Coleoptera: Ciidae) inhabiting the fruiting bodies of Basidiomycetes in North America. - Bull. Mus. Comparative Zool. 145: 163-212.

Neumann, P. \& Ritter, W. 2004: A scientific note on the association of Cychramus luteus (Coleoptera: Nitidulidae) with honeybee (Apis mellifera) colonies. - Apidologie 35: 665-666.

Niemelä, T. 2005: Käävät, puiden sienet. Polypores, lignicolous fungi. - Norrlinia 13: 1 - 320 (in Finnish).

Niemelä, T. \& Kinnunen, J. 2006: Koloveden kansallispuiston kääpäinventointi. - Metsähallitus, Etelä-Suomen luontopalvelut. $19 \mathrm{pp}$.

Rassi, P. (ed.) 1993: Suomen kovakuoriaisten (Coleoptera) frekvenssipisteet 1.1.1960 - 1.1.1990. - Maailman Luonnon Säätiön WWF Suomen Rahaston Raportteja 6: 1-136.

Rassi, P., Alanen, A., Kanerva, T. \& Mannerkoski, I. (eds.) 2001: The 2000 Red List of Finnish species. Ministry of the Environment \& Finnish Environment Institute. $432 \mathrm{pp}$.

Salo, P., Niemelä, T., Nummela-Salo, U. \& Ohenoja, E. (eds.) 2005: Suomen helttasienten ja tattien ekologia, levinneisyys ja uhanalaisuus. - The Finnish Environment 689: 1-526.

Schigel, D. S., Niemelä, T. \& Kinnunen, J. 2006: Polypores of western Finnish Lapland and seasonal dynamics of polypore beetles. - Karstenia 46: 37-64.

Schigel, D.S., Niemelä, T., Similä, M., Kinnunen J. \& Manninen O. 2004: Polypores and associated beetles of the North Karelian Biosphere Reserve, eastern Finland. - Karstenia 44: 35-56.

Schigel, D.S. \& Toresson, H.G. 2005: New records of Polyporus pseudobetulinus, a rare polypore fungus (Basidiomycota, Aphyllophorales) in Scandinavia, and notes on associated beetles. - Memoranda Soc. Fauna Flora Fennica 81: 102-107.

Silfverberg, H. 2004: Enumeratio nova Coleopterorum Fennoscandiae, Daniae et Baltiae. - Sahlbergia 9: $1-111$.

Yakovlev, E. \& Hokkanen, T. J. 1995: Cyllodes ater (Coleoptera, Nitidulidae) found again in Finland. - Entomol. Fennica 5: 203-204.

Zaitsev, A.I. \& Kompantsev, A.V. 1987: Complexes of Coleoptera and Diptera, associated with carpophores of wood-rotting fungi of genus Pleurotus (Fr.) Quél. in Eastern Siberia and Far East. [Комплексы жесткокрылых и двукрылых насекомых, связанных с карпофорами древоразрушающих грибов рода Pleurotus (Fr.) Quél. в Восточной Сибири и на Дальнем Востоке]. In: Ecology and morphology of insects-inhabitants of fungal substrates. [Экология и морфология насекомых-обитателей грибных субстратов]. - Moscow, Nauka: 56-65. (In Russian). 